\title{
Determinación de las Propiedades de Textura de Tabletas de Chocolate Mediante Técnicas Instrumentales
}

\author{
Armando Alvis*, Luis Pérez y Guillermo Arrazola \\ Universidad de Córdoba, Departamento de Ingeniería de Alimentos, Facultad de Ciencias \\ Agrícolas, Carrera 6 No 76-103, Km 3, vía Cereté, Córdoba-Colombia. (e-mail: \\ aalvis2@hotmail.com)
}

${ }^{*}$ Autor a quien debe ser dirigida la correspondencia

Recibido Oct. 01, 2010; Aceptado Nov. 22, 2010; Versión Final recibida Nov. 26, 2010

\begin{abstract}
Resumen
Se han elaboraron diferentes formulaciones de tabletas de chocolate amargo, chocolate blanco y de chocolate con leche, usando la norma NEIAL-6737: 2006. Se realizó una evaluación de la dureza a 2 y $4 \mathrm{~mm}$ de penetración, consistencia "K" calculada a partir del ajuste del modelo de Mottram, esfuerzo de fractura y modulo elástico. Los resultados muestran que para la dureza existen diferencias significativas $(p \leq 0,05)$ entre la tableta amarga y la blanca. Los valores de la consistencia fueron significativamente diferentes $(p \leq 0,05)$ indicando que existen diferencias de textura entre las tabletas de chocolate amarga, blanca y de leche. Para la fracturabilidad, las tabletas amarga y blanca no presentaron diferencias significativas $(p \leq 0,05)$ entre sí en cuanto a esfuerzo de fractura ni módulo elástico. Pero estas a su vez, se fracturan a esfuerzos significativamente menores $(p \leq 0,05)$ que las tabletas de leche.
\end{abstract}

Palabras claves: chocolate, esfuerzo de fractura, penetración, dureza, propiedades de textura

\section{Determination of Textural Properties of Chocolate Tablets by Instrumental Techniques}

\begin{abstract}
Different formulations of tablets of bitter chocolate, white and milk, based on standard NEIAL 6737: 2006, have been manufactured and evaluated. Hardness at 2 and $4 \mathrm{~mm}$ of penetration, consistency $\mathrm{K}$ calculated from Mottram's model, fracture stress and elastic modulus were determined. The results show that for hardness, significant differences $(p \leq 0.05)$ between the bitter pill and white were found. The values of consistency $K$ were significantly different $(p \leq 0.05)$ indicating that there are differences in texture between the bitter chocolate bars, white and milk. For fracture, bitter and white tablets showed no significant differences $(p \leq 0.05)$ between them in terms of fracture stress or elastic modulus. But these, in turn, are fractured with significantly lower efforts than milk bars.
\end{abstract}




\section{INTRODUCCION}

El chocolate es en esencia, una masa de partículas secas de cacao y azúcar finamente dividida suspendida en manteca de cacao. Cada manteca de cacao tiene una textura distinta, debido a las interacciones únicas de polimorfismo entre las estructuras de los lípidos. El chocolate tiene una propiedad única de sensación en la boca debido a que la manteca de cacao tiene un punto de fusión bajo, muy cercano a la temperatura del cuerpo. El tamaño de las partículas de chocolate es también extremadamente importante en la sensación suave de la muestra en la boca, lo cual es parte del atractivo de este producto (Prindiville et al., 2000; Andrae-Nightingale et al., 2009).

En cualquier fórmula de chocolate, la grasa es el componente que mantiene al sistema como un todo y son las propiedades de la misma, modificadas en alguna medida por las propiedades de la fase sólida, las que determinan el comportamiento tecnológico, las propiedades físicas, reológicas y de aceptación del chocolate. Además, es la estructura física de la fase grasa es la responsable del brillo, dureza, fracturabilidad, estabilidad al calor, sensación en la boca, liberación del aroma y en general de la satisfacción del consumidor (Jorge et al. 1999, Afoakwa et al., 2007).

Las características texturales del chocolate definen el producto y su durabilidad y en esencia, el estudio de cualquier cambio de materia prima, condiciones de procesamiento, almacenamiento etc., lleva implícito necesariamente la evaluación de la textura Jorge et al. (1999); de allí, la importancia de buscar métodos instrumentales para evaluar una característica tan determinante, ya que medir de forma instrumental los parámetros definitorios de la textura del chocolate, resulta de gran interés práctico y tecnológico. Tscheuschner y Markov (1989), utilizaron una prueba de compresión entre platos paralelos en una maquina Instron y calcularon la fuerza de compresión, los autores encontraron magnificas correlaciones de este parámetro instrumental con la firmeza evaluada sensorialmente. Jorge et al. (1999) trabajaron con una maquina Instron demostraron que en una prueba de flexión de barras de chocolate, bajo determinadas condiciones de operación, se obtienen correlaciones altamente significativas entre la fuerza de fractura y la fragilidad o fractura evaluada sensorialmente por el "snap" o sonido al partir. Andrae-Nightingale et al. (2009) utilizando una sonda cilíndrica y una prueba de doble compresión, obtuvieron correlaciones altamente significativas con la medición sensorial de la textura de barras en miniatura de chocolate.

El presente trabajo tiene como objetivos determinar, mediante técnicas instrumentales, las propiedades de textura de tabletas de chocolate (amargo, blanco y con leche) elaboradas con diferentes formulaciones en la planta de cereales del Instituto de Investigaciones para la Industria Alimentaria (IIIA) utilizando un texturómetro de la firma Stable Micro Systems y realizar una comparación estadística de los parámetros calculados para establecer diferencias texturales entre formulaciones.

\section{MATERIALES Y MÉTODOS}

Para la evaluación de textura a las tabletas de chocolate amargo, chocolate blanco y de chocolate con leche, se utilizaron barras rectangulares con dimensiones 1,6 x 8,1 x 0,9 cm (ancho, largo y alto) elaboradas con diferentes formulaciones según la norma NEIAL - 6737: 2006 adoptada en la Escuela Latinoamericana y del Caribe de Chocolatería del IIIA.

Se tomaron 7 producciones diferentes de tabletas de chocolate elaboradas con distintas formulaciones (chocolate blanco, chocolate con leche y chocolate amargo), que incluían entre sus ingredientes licor de cacao, manteca de cacao, leche entera y descremada en polvo, azúcar refina, vainilla como aromatizante y lecitina de soya como emulsionante y estabilizante para mejorar la textura. El proceso tecnológico de elaboración de las tabletas inició con la recepción, calculo y pesado de los ingredientes, mezclado de las pastas de chocolate, luego se procedió al moldeo, vibrado y enfriamiento, para finalmente desmolderla y envasarla y posteriormente realizarle el análisis de textura. En todos los casos, los estudios de las propiedades de textura se hicieron utilizando el texturómetro TA-HDplus de la firma Stable Micro Systems (SMS) y los cálculos, mediante el software Texture Exponent TEE 32. Cada ensayo se llevó a cabo con 5 réplicas a la temperatura de $20^{\circ} \mathrm{C}$. 
La prueba de penetración con cono, se realizó de forma uniaxial con un cono de $\alpha=30^{\circ}$ sobre el centro de la pieza a la velocidad de $1,6 \mathrm{~mm} / \mathrm{s}$ hasta una profundidad de $6,5 \mathrm{~mm}$, tomando las lecturas de fuerza de penetración a 2 y 4 a $\mathrm{mm}$ de profundidad. Los datos de las curvas de fuerza (F) vs distancia (p), se ajustaron al modelo exponencial de Mottram (1961) para calcular los parámetros $\mathrm{K}$ (consistencia) y $\mathrm{n}$ (exponente de potencia), según la expresión: $\mathrm{F}=\mathrm{K} p^{n}$.

La prueba de fractura en puente se llevó a cabo, colocando la barra de chocolate, sobre el puente de flexión con una abertura de $6 \mathrm{~cm}$ y se ejerció una fuerza en el centro a una velocidad de 1,6 $\mathrm{mm} / \mathrm{s}$ hasta fractura. Se midió la fuerza de fractura como el pico máximo y la distancia a la que fracturó la tableta. Estos datos se convirtieron en esfuerzo de fractura $\left(\sigma_{\text {fract }}\right)$, deformación aparente $(\varepsilon)$ y módulo elástico aparente $(E)$, mediante las ecuaciones:

$$
\begin{aligned}
& \sigma_{\text {fract. }}=\frac{3 F L}{2 b h^{2}} \\
& \mathrm{E}=\frac{F L^{3}}{4 d b h^{3}} \\
& \varepsilon=\frac{6 h d}{L^{2}}
\end{aligned}
$$

Donde: $\mathrm{L}=$ Ancho del puente, $\mathrm{b}=$ Ancho de la muestra, $\mathrm{h}=$ Altura de la muestra, $\mathrm{F}=$ Fuerza máxima, $d=$ Distancia a la que fractura la tableta

Los parámetros calculados, se procesaron de acuerdo a un análisis de varianza de clasificación simple. En los casos que se encontraron diferencias significativas se procedió a comparar las medias por el test de rangos múltiples de Duncan con un $5 \%$ de probabilidad de error y para ello se utilizó el paquete estadístico PASW Statistic 18 (SPSS Inc., 2009)

\section{RESULTADOS Y DISCUSIÓN}

Una curva típica de penetración con cono obtenida por el texturometro se muestra en la figura 1. En ella se indica los datos de fuerza en función de la distancia ajustados al modelo de Mottram (1961) para el cálculo de la fuerza de penetracion a 2 y a $4 \mathrm{~mm}$ y calcular los parametros $\mathrm{K}$ y $\mathrm{n}$.

Las curvas obtenidas mediante el ajuste del modelo corresponden a líneas rectas con intercepto en el eje $\mathrm{Y}$, tal como se aprecia en la figura 2:

En la tabla 1 se presentan los resultados del análisis estadístico realizado a los parámetros calculados a partir de la prueba de penetración con cono de $\left(\alpha=30^{\circ}\right)$. En ella se aprecia que lo valores de $F_{2 \mathrm{~mm}}$ y $F_{4 \mathrm{~mm}}$ para la tableta de chocolate amargo son significativamente superiores ( $p \leq$ $0,05)$ a la de chocolate blanco y que esta fuerzas de penetración no son diferentes entre la tableta de chocolate amargo y la de chocolate con leche así como la de chocolate blanco y la de chocolate con leche, encontrándose aquella de chocolate con leche en valores intermedios entre las otras dos formulaciones. Los valores de $\mathrm{K}$ mostraron la existencia de diferencias significativas $(p \leq 0,05)$ entre todas las muestras por lo que se podrían considerar más discriminativos entre comportamientos que los valores de fuerza aislados, tal como se esperaba. Jorge et al. (1999), demostraron que el valor de consistencia "K" está íntimamente ligado a la dureza evaluada por técnicas sensoriales, por lo que puede decirse que la dureza entre los productos evaluados es diferente, ya que las tabletas tipo chocolate amargo es la más dura de todas, seguida de la de chocolate blanco y de la de chocolate con leche. Esto se debe al efecto positivo de ablandamiento y de disminución de viscosidad que produce la grasa láctea sobre las masas de chocolate que la poseen (Jorge, 1999). 


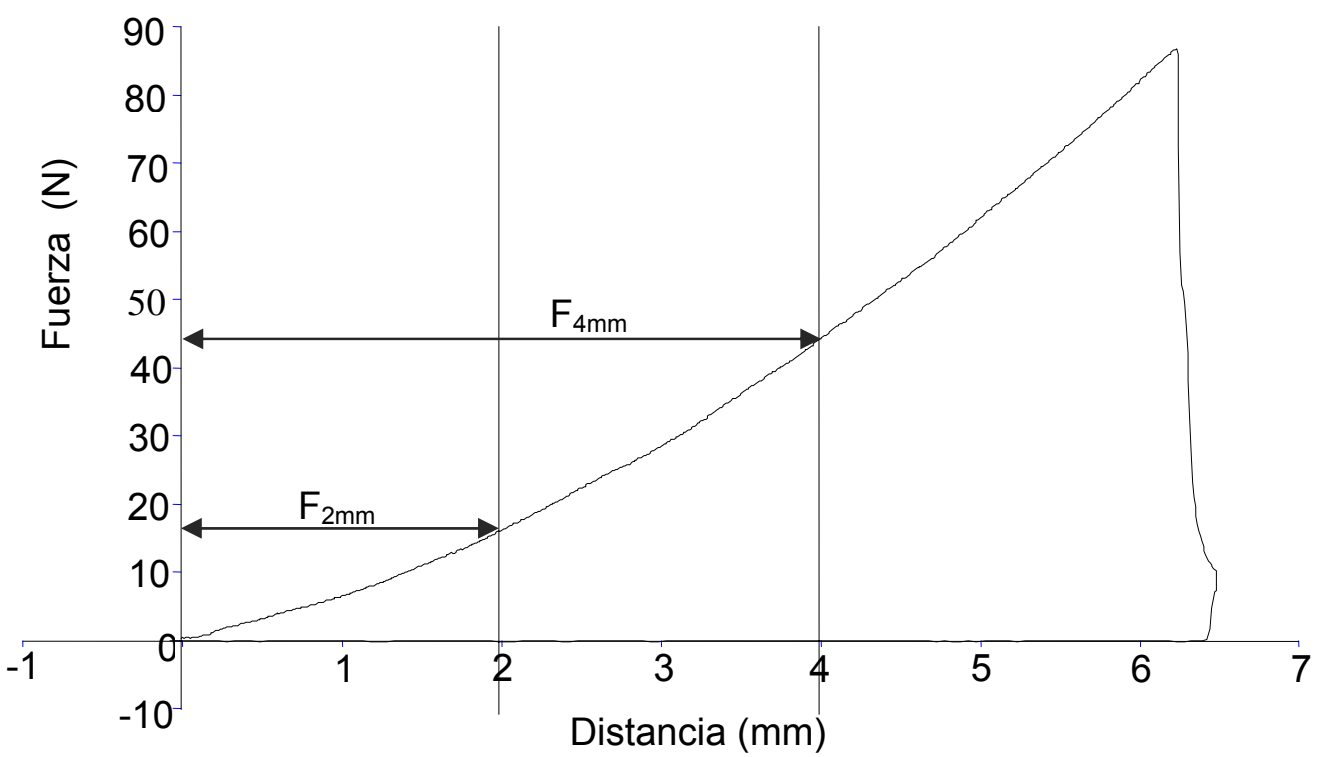

Fig. 1: Curva típica de penetración con cono de $30^{\circ}$

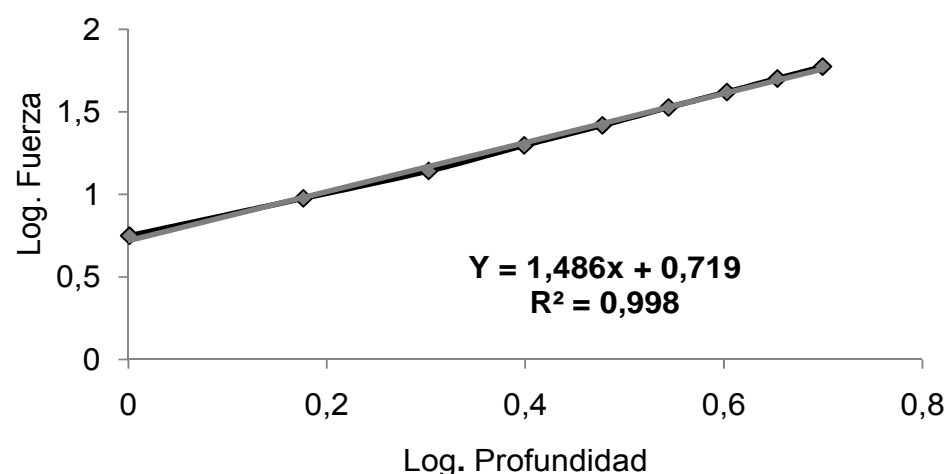

Fig. 2: Ajuste de la curva al modelo de Mottran

Para el exponente de potencia ( $n)$, no se encontraron diferencias significativas $(p \leq 0,05)$, ya que los valores calculados se encontraron entre 1,33-1,38 y se acercaron a los reportados por Jorge et al. (1999) $(n=1,4)$

Tabla 1: Resultados de la prueba de penetración.

\begin{tabular}{|c|c|c|c|}
\hline \multirow{2}{*}{ Parámetros calculados } & \multicolumn{3}{|c|}{ TIPO DE TABLETA } \\
\cline { 2 - 4 } & AMARGO & BLANCO & CON LECHE \\
\hline Fuerza de penetración a $2 \mathrm{~mm}(\mathrm{~N})$ & $15,0^{\mathrm{a}}(0,7)$ & $12,8^{\mathrm{b}}(0,9)$ & $13,7^{\mathrm{a}, \mathrm{b}}(1,3)$ \\
\hline Fuerza de penetración a 4 mm (N) & $38,3^{\mathrm{a}}(1,9)$ & $33,2^{\mathrm{b}}(2,5)$ & $34,9^{\mathrm{a}, \mathrm{b}}(3,3)$ \\
\hline Consistencia “K" (Nmm $\left.{ }^{-\mathrm{n}}\right)$ & $6,36^{\mathrm{a}}(0,08)$ & $4,63^{\mathrm{b}}(0,08)$ & $5,65^{\mathrm{c}}(0,12)$ \\
\hline Exponente "n" (--) & $1,33^{\mathrm{a}}(0,05)$ & $1,34^{\mathrm{a}}(0,06)$ & $1,38^{\mathrm{a}}(0,13)$ \\
\hline
\end{tabular}

$\mathrm{n}=7$. Valores entre paréntesis indican desviación típica. Letras diferentes indican diferencias significativas $p \leq 0,05$.

Full et al. (1996) usando una prueba de penetración con cono $\left(\alpha=10^{\circ}\right)$ en un texturómetro TA$\mathrm{XT2i}$, encontró alta correlación $(r=0.96)$ entre la dureza de la tableta de chocolate medida 
instrumental y sensorialmente, además reportó que la firmeza disminuye con la concentración de grasa de leche en la formula. Desde el punto de vista práctico, se pudo comprobar que con el texturómetro TA-HDplus, la determinación de la fuerza de penetración a 2 y a $4 \mathrm{~mm}$, con cono $(\alpha$ $=30^{\circ}$ ) y una velocidad de $1.6 \mathrm{~mm} / \mathrm{s}$, representa una forma rápida y sencilla de medir la dureza instrumental, en tabletas o cualquier otro producto sólido de chocolate, pues en el caso del parámetro dureza la forma de la muestra bajo estudio no influye, cosa que no sucede con la determinación del esfuerzo de fractura, donde la técnica establecida requiere de unas dimensiones dadas de la barra a analizar (Jorge et al. 1999).

La figura 3 muestra una curva típica para las tabletas de chocolate, obtenida por el puente de flexión y en ella se observa el pico máximo de fractura, necesario para calcular el esfuerzo de fractura y el modulo elástico aparente.

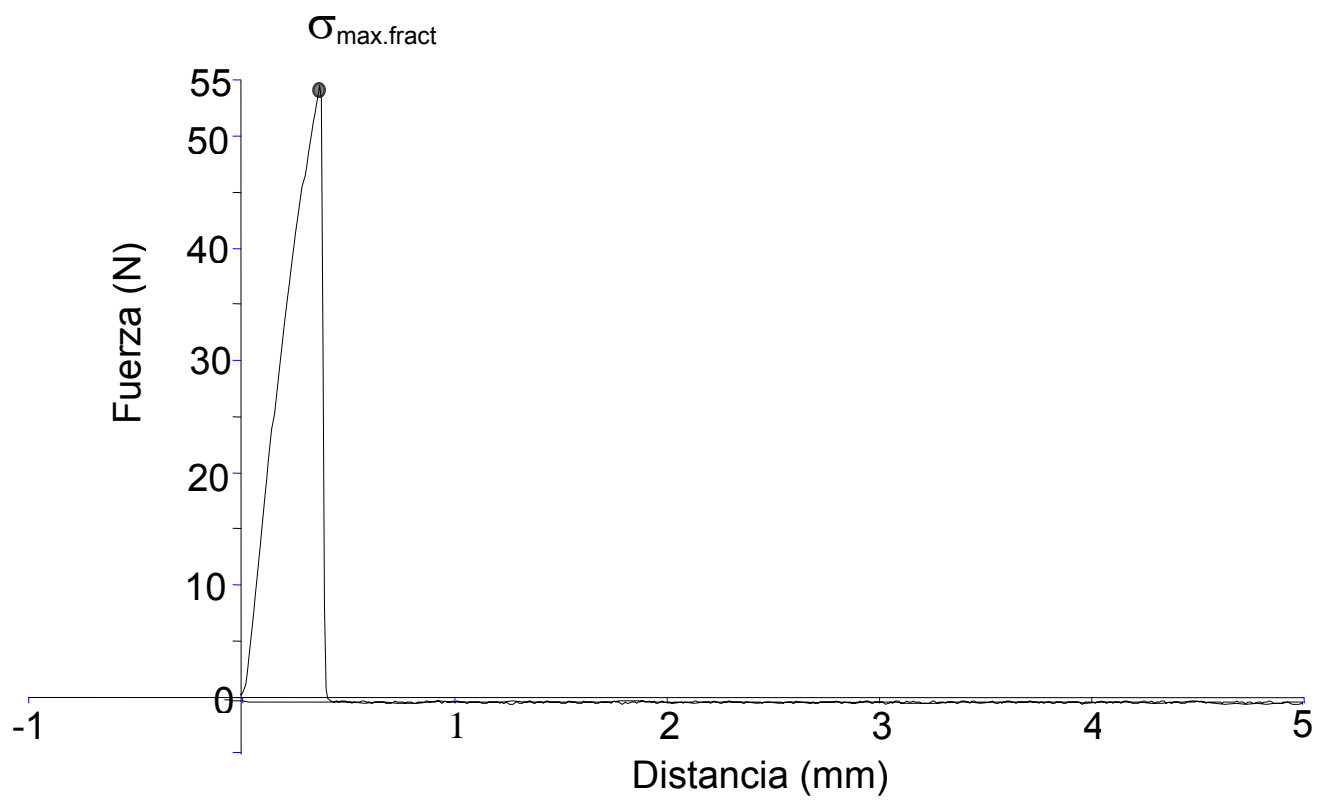

Fig. 3: Curva típica de puente de fractura.

Según los valores reportados en la Tabla 2, la tableta de chocolate con leche presentó el mayor valor de esfuerzo de fractura que fue significativamente más alto que el valor de la tableta de chocolate amargo y de chocolate blanco entre las que no se encontraron diferencias significativas $(p \leq 0,05)$.

Clark y Rao (1978), reportaron para el espagueti seco $\left(E=3^{*} 10^{9} \mathrm{~N} / \mathrm{m}^{2}\right)$ que es un producto firme y fracturable, al igual que para el maíz $\left(E=2,13\right.$ a $\left.6,75^{*} 10^{8} \mathrm{~N} / \mathrm{m}^{2}\right)$, la zanahoria $\left(E=2\right.$ a $\left.4^{*} 10^{7} \mathrm{~N} / \mathrm{m}^{2}\right)$ y la papa $\left(E=0,7\right.$ a $\left.0,92^{*} 10^{7} \mathrm{~N} / \mathrm{m}^{2}\right)$, los cuales se acercan mucho a los valores del modulo elástico calculados para las tabletas de chocolate, que se encuentra en un rango de 3,42 a $4,13^{*} 10^{8} \mathrm{~N} / \mathrm{m}^{2}$ siendo la tableta de chocolate amargo la del menor valor de $\mathrm{E}$ y la de leche la de mayor valor.

Tabla 2. Resultados de la prueba de fractura.

\begin{tabular}{|c|c|c|c|}
\hline \multirow{2}{*}{ Parámetros } & \multicolumn{3}{|c|}{ TABLETAS } \\
\cline { 2 - 4 } & AMARGA & BLANCA & LECHE $^{*}$ \\
\hline$\sigma_{\text {fract }}(\mathrm{kPa})$ & $3552,0^{\mathrm{a}}(148,4)$ & $3678,2^{\mathrm{a}}(209,7)$ & $4050,2^{\mathrm{b}}(150,1)$ \\
\hline $\mathrm{E}(\mathrm{kPa})$ & $342797,0^{\mathrm{a}}(38443,2)$ & $363755,9^{\mathrm{a}, \mathrm{b}}(49038,8)$ & $413601,0^{\mathrm{b}}(48595,5)$ \\
\hline
\end{tabular}

$\mathrm{n}=7$. Valores entre paréntesis indican desviación típica. Letras diferentes indican diferencias significativas $p \leq 0,05$. 
De acuerdo a estos resultados se puede asegurar que las tabletas de chocolate con leche, poseen una red de cristalización del sistema graso fuerte y compacto, ya que según Jorge (1997), el esfuerzo de fractura del producto incrementa en la medida en que haya una mejor cristalización de la grasa presente en la tableta. Además se aprecia que la fracturabilidad está ligada con la dureza y muy seguramente con la cohesividad, pues un producto fracturable tiene una baja cohesividad y una dureza que puede variar de baja a alta Jorge et al. (1999), para este caso la fracturabilidad de las tabletas estuvo inversamente relacionada con la dureza; es decir, la tableta de chocolate amargo que presentó mayores valores de dureza, fue la mas fracturable (requiriendo menor esfuerzo de fractura).

Finalmente, al igual que Jorge et al. (1999), que utilizaron el texturómetro universal Instron, se encontró que tanto para el esfuerzo de fractura como para la fuerza de penetración a 2 y a $4 \mathrm{~mm}$ de profundidad, hay diferencias significativas $(p \leq 0,05)$ entre los valores obtenidos para las tabletas, utilizando el texturómetro TA-HDplus, lo que demuestra que los métodos evaluados son capaces de detectar diferencias texturales en los chocolates y que los valores obtenidos explican además las diferencias reales en textura, pues los chocolates blancos, que no poseen manteca de cacao son los menos duros seguidos por los que contienen grasa láctea dadas las características de cristalización del sistema graso que los forman (Jorge, 1997).

Estos resultados permiten proponer la generalización de estos métodos para la determinación instrumental de la textura en tabletas de chocolate y para emplearse en el control de la calidad y posteriormente en trabajos de investigación y desarrollo.

\section{CONCLUSION}

Las tableta de chocolate elaboradas con pasta amarga presentan mayor dureza que la de chocolate blanco o la elaborada con chocolate con leche y se corrobora que el parámetro K puede ser una característica importante de calidad de estos productos capaz de discriminar entre formulaciones. Las tabletas de chocolate amargo son significativamente más frágiles que las elaboradas con chocolate con leche y también presentan un módulo elástico significativamente menor. Las técnicas y procedimientos desarrolladas en esta investigación, son apropiadas para diferenciar formulaciones de tabletas de chocolate y evaluar sus características más importantes de textura. Se destaca en este trabajo, el cálculo del módulo elástico, como propiedad mecánica fundamental de los alimentos que no ha sido reportada anteriormente para estos productos.

\section{REFERENCIAS}

Afoakwa, E.O., A. Paterson y M. Fowler; Factor influencing rheological and textural qualities in chocolate. Trends in Food Science and Technology 18(6): 290-298 (2007).

Andrae-Nightingale, L., L.Soo-Yeun y N. Engeseth, Textural changes in chocolate characterized by instrumental and sensory techniques. Journal of Texture Studies 40(4): 427-444 (2009).

Clark, R. y V. Rao, Dynamic testing of fresh peach texture. Trans. ASAE 21: 777-781 (1978).

Full, N., S. Yella, P. Dimick, y G. Siegler; Physical and sensory properties of milk chocolate with fat fractions. Journal Food Science. 61(5): 510-518(1996).

Jorge M. Papel de las grasas en las características del chocolate. La manteca de cacao. Material Docente "Avances en la Industria transformadora de Cacao y en la elaboración de chocolate" IIIA. La Habana (Cuba), p 3 - 18 (1997).

Jorge, M., A. Chiralt, y De Hombre, R. Contribución al estudio de la textura del chocolate elaborado con manteca de cacao cubana. Tesis Doctoral en Ciencia y Tecnología de Alimentos. Universidad Politécnica de Valencia (España), p 1 - 125 (1999).

Mottram, F. Evaluation of pseudo-plastic materials by cone penetrometers. Laboratory Practice 10: $767-770$ (1961). 
NEIAL - 6737: Norma de Control de Proceso Productivo de bombones y tabletas macizas. IIIA. La Habana (Cuba), p 2 -11 (2006).

Prindiville, E.A., H. Marshall y H. Heymann; Effect of milk fat, cocoa butter and whey protein fat replacer on the sensory properties of lowfat and notfat chocolate ice cream. Journal of Dairy Science, 83(10): 2216-2223 (2000).

Tscheuschner, H. y E. Markov. Instrumental texture studies on chocolate III. Compositional factors influencing texture. Journal Texture Studies 20: 335-345(1989). 
